\title{
Effect of yoga therapy on fasting blood sugar and to study the distribution of anthropometric measures in type-2 diabetes
}

\begin{abstract}
Diabetes has become one of the most dreaded diseases in the modern world. In the case of India, it has become the diabetes capital of the world. In the future it will increase, making one out of six a diabetic person. Further a majority of the people are not aware that they are diabetic. Diabetes leads to complications like risk of heart attack, kidney failure, stroke, nerve damage etc. Yoga therapy strikes at the root of the problem which is life style. It is greatly useful for pre diabetics. It is cost effective and safe. Studies have brought out the role of yoga therapy in creating positive emotions and in the management of diabetes mellitus. The aim of the study is to assess the effect of SVYASA prescribed yoga module on fasting blood sugar in type 2 diabetes. ${ }^{1,2}$

60 participants with diabetes within the age group of 25-65 in and around Bengaluru city limit namely Yelahanka and Matthikere in Karnataka. All the participants were given the yoga module which included asana, pranayama, relaxation practices, and meditation classes, for a period of two weeks. An introduction to the study, a comprehensive questionnaire, and written informed signed consent was got from all participants at the beginning of the study. Assessments were done using calibrated electronic glucometer. Fasting and postprandial blood sugars were measured before and after yoga intervention. The study also focused on personalization of the yoga module based on profiling of the participants leading to better outcomes as opposed to a standard protocol based therapy.
\end{abstract}

Keywords: type-2 diabetes, obesity, yoga, fasting blood sugar, anthropometric measures, stress
Volume II Issue I - 2018

Samyukta Kudigra,Venkatram, Akanksha NS SVYASA University, India

Correspondence: Samyukta Kudigram, Brigade Palmsprings, Apt A 1003, 24 th Main, JP Nagar $7^{\text {th }}$ Phase, Bangalore, Karnataka, India, Tel 9945448447, Email samyuktasamaga I @gmail.com

Received: December 17,2017 | Published: February 05, 2018
Abbreviations: FBS, fasting blood sugar; DM, diabetes mellitus; IDF, international diabetes federation; WPR, western pacific region; WHO, world health organization; IR, insulin resistance; PSS, perceived stress scale

\section{Introduction}

Diabetes (diabetes mellitus) is a metabolic disorder, where blood sugar levels spike over a prolonged period. According to statistics from the International Diabetes Federation (IDF), India has more diabetics than any other nation of the world. Current estimates peg the number of diabetics in the country at about 62 million. Lifestyle is strongly associated with the development of Type 2 Diabetes, and lifestyle change is the cornerstone of its management. Considering the far-reaching social, psychological and economic costs associated with this condition, extensive research on effectiveness of Yoga therapy on diabetes has already been conducted. The research in this paper restricts itself to determining the effectiveness of S-Vyasa prescribed yoga module for diabetics in managing diabetes, employing the wellknown measurement criteria such as Fasting Blood Sugar.

\section{How are diabetes and obesity related?}

Cardiovascular disease remains the leading cause of death in women. Both obesity and diabetes mellitus are important independent risk factors for the development of cardiovascular disease. Obesity is the leading risk factor for type 2 diabetes. Women with a body mass index (BMI) of $30 \mathrm{~kg} / \mathrm{m}^{2}$ have a 28 times greater risk of developing diabetes than do women of normal weight. The risk of diabetes is 93 times greater if the BMI is $35 \mathrm{~kg} / \mathrm{m} 2$. The national prevalence rates of diabetes have increased in parallel with the rates of obesity. The best treatment for diabetes is prevention. Prevention of diabetes can be accomplished through a $7 \%$ weight loss through intensive lifestyle interventions that include caloric reduction and approximately $30 \mathrm{~min}$ of daily moderate physical activity. (The Epidemic of Obesity and Diabetes-Trends and Treatments by Ann Smith Barnes, MD, MPH Stephanie A. Coulter, MD, Section Editor). Current literature suggests a high burden of generalized obesity among Indian children and adolescents, with a definite socioeconomic gradient. AsianIndian children are increasingly susceptible to unfavourable body composition, as well as regional adiposity. The conventional BMI criteria for obesity are inadequate to identify these differences in body fat composition or distribution. Hence, ethnicity-specific, metabolically relevant cut-off values should be considered while diagnosing obesity and adiposity. Available evidence on the natural history of type 2 diabetes in India suggests the need for a lifecourse approach in the prevention and control of childhood obesity. A comprehensive multilevel, multicomponent obesity-prevention strategy addressing a wide range of issues, starting from maternal and childhood undernutrition, and including sociodemographic and environmental factors, is a necessity in India. (Childhood obesity and type 2 diabetes in India by Pradeep A Praveen, Nikhil Tandon). 


\section{Diabetes and patient education}

There was a study that investigated the influence of a number of psychological factors upon the practice and outcome of diabetic selfmanagement among young adults with insulin-dependent diabetes mellitus. The results demonstrated that the sample members perceived that they were responsible for the control of their diabetes, that the benefits of following treatment were greater than any barriers and that they were knowledgeable about their diabetes Vivien E Coate et al. ${ }^{3}$ Patients' adherence, attitude, beliefs, and knowledge about diabetes may affect diabetes self-management. Culture and language capabilities influence the patient's health beliefs, attitudes, health literacy, thereby affecting diabetes self-management Soohyun Nam et $\mathrm{al}^{4}{ }^{4}$ In another study, randomized control trial study to improve diabetes self-management through acceptance, mindfulness, and values was conducted. It was observed that after three months there was improvement in their coping skills acceptance coping and selfmanagement behavior mediated the impact of treatment on changes in $\mathrm{HbA} 1 \mathrm{c}$ Jennifer AG, et al. in 2012. Another study showed that subjects characterized by strong beliefs in their self-efficacy and an optimistic outlook on life were more likely to be satisfied with their doctor-patient relationships. They demonstrated more active coping behavior and proved to have a higher quality of life. Active coping behavior was the only psychological variable significant for the HbAlc values Matthias Rose et al. ${ }^{5}$

\section{Diabetes and social support}

Adults with type 2 diabetes $(\mathrm{N}=119 ; 29 \%$ Latino, 61\% Black, $25 \%$ White) completed validated measures of diabetes distress and social support. Multiple linear regression evaluated the moderating role of social support in the relationship between diabetes burden, indicated by prescription of insulin and presence of complications, and distress. Findings support the stress-buffering hypothesis and suggest that social support may protect against diabetes distress. Rachel N Baek et al. ${ }^{6}$

\section{Diabetes and yoga}

Yoga is considered as one of the preventive and alternative treatment in diabetes management. Yoga has been studied for controlling both the symptoms and complications associated with Diabetes Mellitus Type 2. The results of the study suggested a statistically significant role of yoga in controlling diabetes. It shows that diabetics may benefit from yoga's ability to improve quality of life, Aljasir et al. ${ }^{7}$ Yoga therapy has been useful in treatment of negative symptoms and improving the socio- occupational functioning and emotion recognition deficits in antipsychotic- stabilized schizophrenia patients, Jayaram et al. ${ }^{8}$ Yoga has proved to be an effective tool as an add-on therapy in chronic depression patients, Naveen et al. ${ }^{9}$ Yoga has also proved its efficacy in psychological determinants such as mood disturbances and stress symptoms in cancer patients Mackenzie et al. ${ }^{10}$ Hatha yoga practices have proved its benefits in stress reduction in middle aged women which is shown by a stress reduction scale called PSS (Perceived Stress Scale), Moliver et al. ${ }^{11}$

The integrated approach of yoga comprising of physical postures, breathing and meditation helped to manage psychological stress and thereby leading to the physical benefits, which is described through the Adhija Vyadhi model. We also found that IYN protocol was easy to adhere to and the results were sustained for 2 months following the discharge of the patient. We plan to follow-up with the patient further to understand the long-term benefits of Yoga and Naturopathy based lifestyle interventions in MetS. This case also adds to growing clinical evidence of the use of Yoga and Naturopathy therapies in chronic non-communicable degenerative disorders. The significant changes we found may be attributed to an integrated approach of Naturopathy and Yoga, as well as the whole hearted adherence of the patient. The case report shows remarkable changes in MetS status that improved the quality of life following a 6 week IYN intervention. The change was sustainable for 12 weeks through simple lifestyle modifications. Considering the effects achieved in this case, IYN could be used as a safe and beneficial intervention to integrate into the management of MetS. Swathi Gowda et al. ${ }^{12}$ A group of 44 accomplished diabetes patients in the age group of 40-55years with diabetes 1 to 10 years were included. Test group and control group were each divided with 22 patients. The test group was taught yoga in the mornings by a yoga expert. The result showed significant decline in metabolic parameters, FBS, PPBS, triglycerides and LDL. Group also showed significant decrease in weight, BMI and waist hip ratio in the test group Balaji, et al. in 2011.

41 participants with high risk type 2 diabetes were randomized in yoga $(\mathrm{n}=21)$ and a walking control (20) in Bangalore, India. Participants were asked to either attend yoga classes or complete monitored walking 3-6days per week for eight weeks. Among people with elevated fasting blood glucose, it was found that participation in an 8 week yoga intervention was feasible and resulted in greater weight loss and reduction in waist circumference when compared to the walking control group Dermott, et al. in 2014. A randomized control trial with the patients having $\mathrm{HbAlc}$ between 6 to $9 \%$ with minimum 3 month history, lifestyle modification and oral and anti-diabetic medication were included. Patients were divided into two groups$1^{\text {st }}$ standard diabetes treatment and $2^{\text {nd }}$ standard diabetes treatment with Sudarshana kriya yoga and Pranayama. There was significant improvement in physical, psychological and social domains, and total QOL post intervention in the group practising the comprehensive yogic breathing program as compared to the group following the standard treatment alone Jyotsna et al. ${ }^{13} 227$ type 2 diabetes patients of both genders aged above 28years in and around Bengaluru were allocated to a yoga based lifestyle modification program and exercise based life style modification program. Medication score, blood glucose, HbA1c and lipid profile were assessed at baseline and after 9months. It showed better reduction in the dosage of hypoglycemic medication required and increase in HDL in the Yoga group as compared to the control group. FBG reduced only in the yoga group Nagarathna, et al. ${ }^{14} \mathrm{~A}$ total of 77 normal healthy individuals and those having hypertension, coronary artery disease, diabetes mellitus and a number of other illnesses were provided yoga for 10days. The outcome measure were subjective wellbeing inventory scores (SUBI) taken on the first and the last days of the course. There was a significant improvement in the subjective wellbeing scores of the 77 subjects in the intervention group within a period of 10 days as compared to the control group Sharma et al. ${ }^{15} \mathrm{~A}$ brief but comprehensive lifestyle modification programs based on yoga for prevention and management of chronic diseases, of 8 days period was conducted. The study found that fasting plasma glucose, cholesterol, etc were lower on the last day of the course, compared to the first day Ramesh L Bijilani, et al. in 2005.

\section{Materials and methods}

Convenient sampling method, with a single group pre-post design was followed, with a sample size of 60 Type-2 diabetic subjects on medication, from the stop diabetes movement camp conducted at Yelahanka and Matthikere localities in Bangalore. The subjects were in the age group of 20-70, both male and female. -All of them were 
willing to take up yogic management of Type 2 diabetes and interested in knowing its effect on the disease. Those on psychiatric medication, physical disabilities and those who had recently undergone any surgeries were excluded from the study.

The S-VYASA prescribed Integrated Yoga module for diabetes, which included the below regimen was used for intervention:

i. Breathing practices: Rabbit breathing, Tiger breathing

ii. Shithilikarana vyayama (loosening exercises): Trikonasana bending, Twisting, Dhanurasana swing, Pavanamuktasana kriya, Surya namaskara, Quick Relaxation Technique

iii. Asanas: Parivrita Trikonasana, Vakrasana/ Ardhamatsyendrasana, Ushtrasana, Bhujangasana, Shalabhasana, Dhanurasana, Sarvangasana, Matsyasana, Deep Relaxation Technique

iv. Bandhas: Uddiyana Bandha, Mula Bandha, Jalandhara Bandha

v. Kriyas: Jala Neti, Sutra Neti, Vamana Dhouthi, Shankha Prakshalana, Agnisara Kriya, Kapalabhati

vi. Pranayama: Vibhagiya Pranayama (Sectional Breathing), Surya Anuloma Pranayama, Nadi Shuddha Pranayama, Sitali Sitakari/Sadanta Pranayama, Brahmari Pranayama

vii. Meditation (Dharana and Dhyana): Nadanusandhana, OM Meditation

Assessment was done using a calibrated electronic glucometer same batch of strips as well as using the same electronic batch chipand fasting blood sugar was collected before and after yoga module intervention. The systolic and diastolic readings were assessed using the standard sphygmomanometer. For measuring the Body Mass Index, weight was measured using the standard weighing scale and height was measured using the standard measuring stick.

Data analysis was done using SPSS version 2.

\section{Results and discussion}

\section{Body mass index}

\begin{tabular}{lll}
\hline Parameter & Mean & Standard deviation \\
\hline BMI & 26.6176 & 4.1565 \\
\hline
\end{tabular}

The mean of 26.6 being greater than 25 , indicates that majority of the participants are overweight.

\section{Waist circumference}

\begin{tabular}{lll}
\hline Parameter & Mean & Standard deviation \\
\hline WC in cms & 67.04386 & 30.95854 \\
\hline
\end{tabular}

As the mean of 67 is less than the thresholds of $94 \mathrm{cms}$ for men and $80 \mathrm{cms}$ for women, the participants do not fall under the high risk category.

\section{Blood pressure}

\begin{tabular}{lll}
\hline Parameter & Mean & Standard deviation \\
\hline BP Systolic & 138.2203 & 21.555116 \\
BP Diastolic & 82.27119 & 8.8275246 \\
\hline
\end{tabular}

The mean of $138 / 82 \mathrm{Hg}$ for the blood pressure in the above table indicates that the participants fell under the low risk category.

\section{Fasting blood sugar}

\begin{tabular}{lcclc}
\hline Parameter & Mean & $\begin{array}{l}\text { Standard } \\
\text { deviation }\end{array}$ & Normality & p-value \\
\hline $\begin{array}{l}\text { FBS Pre } \\
\text { Intervention }\end{array}$ & $164.288 \mathrm{I}$ & 58.09358 & 0.001 & 0.012 \\
$\begin{array}{l}\text { FBS Post } \\
\text { Intervention }\end{array}$ & 155.5392 & 50.64893 & 0.008 & \\
\hline
\end{tabular}

\section{Wilcoxon test}

Wilcoxon Test (FBS Pre and FBS Post) Asymp Sig (2-tailed)

\section{Interpretation of the results of analysis}

The mean of the fasting blood sugar has reduced from 164.2881 to 155.5392 . The standard deviation has also shown a reduction from 58.09358 to 50.64893 . The p-value is 0.012 which is less than 0.05 , which indicates significant difference between pre and post measurements. Physical exercises and yoga practices especially those which give a twist to the vertebral column is very good for diabetic patients as it stimulates the liver and pancreas. Vajrasana, Shashankasana is very good for digestion and diabetic patient. Breathing practices and Pranayama specially cooling practices Sheetali, Sitkari, Sadanta is emphasized which gives a sense of satisfaction and normalizes the increased appetite and thirst. Frequent urination is also controlled as sympathetic and parasympathetic nervous system is balanced. It energizes the whole body and removes tardiness. Bhramari Pranayama soothes and calms down the mind. Cyclic meditation and Nadanusandhana help in reducing stress. ${ }^{16-20}$

\section{Conclusion}

Yoga is effective in reducing fasting blood sugar levels.

\section{Acknowledgments}

The Institute Ethics Committee Approval (SVYASA) was obtained before the starting of the study. The participants were explained the details of the study and their consent was sought to conduct the survey. Each participant who participated in IAYT was also explained in detail about the study and written informed consent was obtained from them before their participation.

\section{Conflict of interest}

The authors declare that they have no conflict of interest in the publication.

\section{References}

1. Yoga Vasishtha.

2. Yoga Philosophy and Health. SVYASA, India; 2011.

3. Coates VE, Boore JR. The influence of psychological factors on the self-management of insulin-dependent diabetes mellitus. J Adv Nurs. 1998;27(3):528-537.

4. Nam S, Chesla C, Stotts NA, et al. Barriers to diabetes management: patient and provider factors. Diabetes Res Clin Pract. 2011;93(1):1-9.

5. Rose M, Fliege H, Hildebrandt M, et al. The network of psychological variables in patients with diabetes and their importance for quality of life and metabolic control. Diabetes Care. 2002;25(1):35-42. 
6. Baek RN, Tanenbaum ML, Gonzalez JS. Diabetes burden and diabetes distress: the buffering effect of social support. Ann Behav Med. 2014;48(2): $145-455$.

7. Aljasir B, Bryson M, Al Shehri B. Yoga Practice for the Management of Type II Diabetes Mellitus in Adults: A systematic review. Evid Based Complement Alternat Med. 2010;7(4):399-408.

8. Jayaram, Varambally S, Behere RV, et al. Effect of yoga therapy on plasma oxytocin and facial emotion recognition deficits in patients of schizophrenia. IJP. 2013;55(7):409-413.

9. Naveen GH, Thirthalli J, Rao MG, et al. Positive therapeutic and neurotropic effects of yoga in depression: A comparative study. Indian $J$ Psychiatry. 2013;55(Suppl 3):S400-S404.

10. Mackenzie MJ, Carlson LE, Ekkekakis P, et al. Affect and Midfulness as predictors of change in mood disturbance, stress symptoms and Quality of Life in a community based Yoga program for cancer survivors Evidence-Based Complementary and Alternative Medicine. 2013.

11. Moliver, Mika, Hartrand M, Haussman R. Quality of life and mental health in patients with chronic diabetes who regularly practice Yoga and those who do not; a case control study. Evidence-Based Complementary and Alternative Medicine. 2013.

12. Gowda S, Mohanty S, Saoji A, et al. Integrated Yoga and Naturopathy module in management of Metabolic Syndrome: A case report. $J$ Ayurveda Integr Med. 2017;8(1):45-48.
13. Jyotsna VP, Joshi A, Ambedkar S, et al. Yogic Breathing improves quality of life in patients with diabetes. Indian $J$ Endocrinol Metab. 2012;16(3):423-428.

14. Cui J, Yan JH, Yan LM, et al. Effects of yoga in adults with type 2 diabetes mellitus: A meta-analysis. J Diabetes Investig. 2017;8(2):201-209.

15. Bijlani RL, Vempati RP, Yadav RK, et al. A brief but comprehensive lifestyle education program based on yoga reduces risk factors for cardiovascular disease and diabetes mellitus. J Altern Complement Med. 2005;11(2):267-274.

16. Shrimad Bhagavad Gita commentary.

17. Gard T, Noggle JJ, Park CL, et al. Potential self-regulatory mechanisms of yoga for psychological health. Front Hum Neurosci. 2014;30(8):770.

18. J Simonds, D Goldstein, B Walker, et al. The Relationship Between Psychological Factors and Blood Glucose Regulation in Insulkidependent Diabetic Adolescents. Diabetes Care. 1981;4(6):610-615.

19. Type 2 diabetes mellitus. Diapedia Collective, India.

20. http://care.diabetesjournals.org 